\title{
Über soziale Verwundbarkeit, Planungsmethoden und Arbeitskräftemobilität
}

\author{
Heiderose Kilper ${ }^{1} \cdot$ Andreas Klee $^{2}$
}

Online publiziert: 28. November 2018

(c) Die Herausgeber 2018

Drei Beiträge aus der raum- und planungswissenschaftlichen Forschung, ein Bericht aus der Praxis sowie drei Rezensionen beschließen das Jahr 2018 der Zeitschrift „Raumforschung und Raumordnung I Spatial Research und Planning“.

Den Auftakt macht der Beitrag „Risikomanagement ohne Risikominderung? Soziale Verwundbarkeit im Wiederaufbau nach Hochwasser in Deutschland" von Mark Kammerbauer (Hochschule Augsburg) und Christine Wamsler (Lund University Centre for Sustainable Studies/LUCSUS). Sie greifen den raumbezogenen ResilienzDiskurs auf und bereichern ihn mit empirischen Befunden aus einer Fallstudie sowie um Empfehlungen für die Planungspraxis. Theoretisch verortet im Sozialkonstruktivismus wird das „Konzept der sozialen Verwundbarkeit“, das heißt der Zusammenhang zwischen Berufsstand, sozialer Stellung, Geschlecht, Alter, Gesundheit, Ethnie etc. und individuell unterschiedlichen Möglichkeiten beim Zugang zu Ressourcen im Kontext von Umwelt- und Klimakatastrophen, entfaltet. Dargestellt wird eine Anwendung des Konzepts in einer empirischen Fallstudie über Planung und Durchführung des Wiederaufbaus von Wohnhäusern in der niederbayerischen Stadt Deggendorf nach dem Extremhochwasser der Donau im Frühsommer 2013. Die Autoren bewerten diesen ex post als Wiederaufbau im Sinne eines „Ersatzes entstandener Schäden“. Sie beleuchten die strategische Bedeutung des „Konzepts der sozialen Verwundbarkeit" für einen Wiederaufbau und sehen darin eine „Weiterentwicklung der bestehenden Siedlungsstruktur“,

Prof. Dr. Heiderose Kilper

heiderose.kilper@leibniz-irs.de

Prof. Dr. Andreas Klee

klee@arl-net.de

1 Leibniz-Institut für Raumbezogene Sozialforschung, Flakenstraße 29-31, 15537 Erkner, Deutschland

2 Akademie für Raumforschung und Landesplanung, Leibniz-Forum für Raumwissenschaften, Hohenzollernstraße 11, 30161 Hannover, Deutschland quasi eine künftige Risikominderung. Daraus formulieren sie (Handlungs)empfehlungen für Verantwortliche in der Planung, im Katastrophenmanagement wie auch für die Öffentlichkeit.

Franziska Ganesch (Otto-Friedrich-Universität Bamberg) stellt in ihrem Beitrag „Regional Mobility and Spatial Inequality" die Frage, aus welchen Gründen insbesondere jüngere Menschen in Deutschland ihre Heimatregionen verlassen, um einer Beschäftigung woanders nachzugehen. Kann dies immer als eine einseitig gerichtete Abwanderung von Fachkräften aus strukturschwachen Räumen interpretiert werden, wie es beispielsweise Presseberichte und TVReportagen gern suggerieren? Mittels logistischer Regressionsmodelle kann Franziska Ganesch nachweisen, dass die altersselektive Abwanderung aus ökonomisch schwachen Regionen zwar eine große Bedeutung hat, aber allein betrachtet - das beschäftigungsbezogene Wanderungsverhalten nicht vollständig erklären kann. Denn das jeweilige Bildungsniveau und das Lebensalter erklären das Wanderungsgeschehen ebenfalls - und dies sowohl in strukturstarken wie strukturschwachen Regionen. Ihr Beitrag ist somit als Plädoyer zu verstehen, die regionalen, raumstrukturellen Bedingungen nicht überzubewerten, sondern regionale Disparitäten auch im Lichte sich ändernder individueller beruflicher Qualifikationen zu diskutieren.

Befunde aus dem DFG-Forschungsprojekt „Raumplanungsmethoden als kollektive Handlungsressourcen im Communicative Turn" präsentieren Christian Diller (Justus-Liebig-Universität Gießen) und Sarah Oberding (Kreisverwaltung Lahn-Dill-Kreis) in ihrem Beitrag , ,Rationale“ vs. (?) ,kommunikative" Planungsmethode: Theoretische Ausgangspunkte, empirische Befunde aus Experimenten und Überlegungen zur Weiterentwicklung am Beispiel der Nutzwertanalyse“. Das Fragezeichen im Titel des Beitrags deutet es bereits an: Die Untersuchung hat aus Sicht der Autoren das Ergebnis erbracht, dass die Trennung zwischen rationalen, kommunikativen und kreativen Planungsmethoden allenfalls eine graduelle ist. Als entscheidend wird deren Performanz danach gewertet, inwieweit es ihnen gelingt, im jeweiligen institutionellen und praktisch-planeri- 
schen Kontext die Potenziale von Akteuren zu mobilisieren. Der Beitrag endet mit dem Plädoyer, in künftigen planungstheoretischen Debatten die kreativ-emotionale Dimension planerischer Methoden stärker einzubeziehen.

Unter der Rubrik „Bericht aus der Praxis“ stellen Dirk Wittowsky und Florian Ahlmeyer (beide ILS - Institut für Landes- und Stadtentwicklungsforschung, Dortmund) in ihrem Beitrag ,Was brauchen wir in ländlichen Räumen? Erreichbarkeitsmodellierung als strategischer Ansatz der regionalen Standort- und Verkehrsplanung“ ein integriertes Raum- und Verkehrsplanungswerkzeug vor, das die ,Erreichbarkeit“" von Standorten der Daseinsvorsorge aus der Position der Nachfrager bemisst. Das Tool ist im Rahmen des Projekts „Erreichbarkeitssicherung im ländlichen Raum“ entwickelt worden, gemeinsam mit dem Institut für Straßen- und Verkehrswesen der Universität Stuttgart. Finanziert wurde das Vorhaben vom Ministerium für ländlichen Raum und Verbraucherschutz des Landes BadenWürttemberg. Untersuchungsgebiet und damit Modellraum war der Landkreis Calw am nördlichen Schwarzwaldrand, der unterschiedliche Gegebenheiten des ländlichen Raums repräsentiert. Dem vor Ort entwickelten Raum- und Verkehrsplanungswerkzeug bescheinigen die Autoren mehrere Vorteile: Transparenz, weil auf der Grundlage nachprüfbarer Daten Defizite in der Erreichbarkeit von Standorten der Daseinsvorsorge berechnet und visualisiert werden können; Flexibilität und Dynamik, weil dies sowohl für den Status quo als auch für künftige Bevölkerungs- und Angebotsver- änderungen im Öffentlichen Personennahverkehr gilt; sachliche Adäquanz, weil es der Heterogenität ländlicher Räume gerecht wird.

In Heft 4.2018 haben wir Sie bereits darüber informiert, dass ab Jahresanfang 2019 die Zeitschrift „Raumforschung und Raumordnung I Spatial Research and Planning“ als Open-Access-Zeitschrift (Goldener Weg) erscheinen wird, das heißt, es wird parallel die Bereitstellung als Print- und als digitale Erstveröffentlichung erfolgen. Damit verbunden ist ein Wechsel zum Verlag De Gruyter Open, einem der führenden wissenschaftlichen Verlage im Open-AccessModell.

Auch bei Open Access publizieren die Autorinnen und Autoren in einer referierten Fachzeitschrift. Das etablierte doppelblinde Review-Verfahren wird unverändert beibehalten, ebenso das Qualitätssicherungsverfahren durch die Schriftleiter und das Lektorat. Das Procedere der Manuskripteinreichung und des Review-Verfahrens über ein webbasiertes Managementsystem bleibt ebenfalls im Wesentlichen unverändert.

Wir danken Ihnen für Ihr Interesse, das Sie als Leser und Autoren der Zeitschrift „Raumforschung und Raumordnung I Spatial Research and Planning“ entgegengebracht haben, und wünschen Ihnen erholsame Weihnachtstage und einen guten Start in das neue Jahr 2019.

Heiderose Kilper und Andreas Klee 